\title{
Antimicrobial resistance of major clinical pathogens in South Korea, May 2016 to April 2017: first one-year report from Kor-GLASS
}

Hyukmin Lee ${ }^{1,2}$, Eun-Jeong Yoon ${ }^{1,2}$, Dokyun Kim¹, Seok Hoon Jeong¹, Eun Jeong Won³, Jong Hee Shin ${ }^{3}$, Si Hyun Kim4, Jeong Hwan

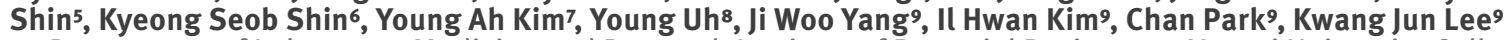

1. Department of Laboratory Medicine and Research Institute of Bacterial Resistance, Yonsei University College of Medicine, Seoul, Republic of Korea

2. These authors contributed equally to this study

3. Department of Laboratory Medicine, Chonnam National University School of Medicine, Gwangju, Republic of Korea

4. Department of Clinical Laboratory Science, Semyung University, Chungbuk, Republic of Korea

5. Department of Laboratory Medicine and Paik Institute for Clinical Research, Inje University College of Medicine, Busan, Republic of Korea

6. Department of Laboratory Medicine, Chungbuk National University College of Medicine, Cheongju, Republic of Korea

7. Department of Laboratory Medicine, National Health Insurance Service Ilsan Hospital, Goyang, Republic of Korea

8. Department of Laboratory Medicine, Yonsei University Wonju College of Medicine, Wonju, Republic of Korea

9. National Institute of Health, Centers of Disease Control and Prevention, Cheongju, Republic of Korea

Correspondence: Seok Hoon Jeong (kscpjsh@yuhs.ac)

Citation style for this article:

Lee Hyukmin, Yoon Eun-Jeong, Kim Dokyun, Jeong Seok Hoon, Won Eun Jeong, Shin Jong Hee, Kim Si Hyun, Shin Jeong Hwan, Shin Kyeong Seob, Kim Young Ah, Uh Young, Yang Ji Woo, Kim II Hwan, Park Chan, Lee Kwang Jun. Antimicrobial resistance of major clinical pathogens in South Korea, May 2016 to April 2017 : first oneyear report from Kor-GLASS. Euro Surveill. 2018;23(42):pii=1800047. https://doi.org/10.2807/1560-7917.ES.2018.23.42.1800047

The Korean government established an antimicrobial resistance (AMR) surveillance system, compatible with the Global AMR Surveillance System (GLASS): Kor-GLASS. We describe results from the first year of operation of the Kor-GLASS from May 2016 to April 2017, comprising all non-duplicated clinical isolates of major pathogens from blood,urine, faeces and urethral and cervical swabs from six sentinel hospitals. Antimicrobial susceptibility tests were carried out by disk diffusion, Etest, broth microdilution and agar dilution methods. Among 67,803 blood cultures, 3,523 target pathogens were recovered. The predominant bacterial species were Escherichia coli $(\mathrm{n}=1,536)$, Klebsiella pneumoniae $(\mathrm{n}=$ 597) and Staphylococcus aureus $(\mathrm{n}=584)$. From 57,477 urine cultures, $6,394 \mathrm{E}$. coli and 1,097 K. pneumoniae were recovered. Bloodstream infections in inpatients per 10,000 patient-days (10TPD) were highest for cefotaxime-resistant $E$. coli with 2.1 , followed by 1.6 for meticillin-resistant Sta. aureus, 1.1 for imipenem-resistant Acinetobacter baumannii, 0.8 for cefotaxime-resistant $K$. pneumoniae and 0.4 for vancomycin-resistant Enterococcus faecium. Urinary tract infections in inpatients were 7.7 and 2.1 per 10TPD for cefotaxime-resistant $E$. coli and $K$. pneumoniae, respectively. Kor-GLASS generated well-curated surveillance data devoid of collection bias or isolate duplication. A bacterial bank and a database for the collections are under development.

\section{Introduction}

Antimicrobial resistance (AMR) is a growing burden in both clinical and socioeconomic context owing to the high morbidity and prolonged hospitalisation of patients that causes elevated medical and societal costs because of loss of productivity [1]. The World Health Organization launched the Global AMR Surveillance System (GLASS) in 2015 [2] as a core global action plan addressing this issue. The standardised GLASS manual allowed an overview of global AMR rates through international comparison.

An AMR surveillance system in South Korea, the Korean AMR Monitoring System (KARMS), had been operated between 2002 and 2015 by Korean Centers for Disease Control and Prevention (KCDC) [3,4]. KARMS played an important role in notifying the high AMR rates in South Korea, urging the government to develop a national action plan. However, this system had limitations. Firstly, the antimicrobial susceptibility testing (AST) methods were not well harmonised across the participating clinical laboratories, affecting reliability. Secondly, duplicated isolates were not sufficiently filtered out, which could lead to an overestimation of the national AMR rates. Finally, the epidemiological interpretation of the study was limited because of insufficient clinical data.

From KARMS, the KCDC established an improved AMR surveillance system compatible with the GLASS, named Kor-GLASS [5]. The Kor-GLASS manual was customised 


\section{FIGURE 1}

Number of patients sampled for bacterial culture, by specimen and age group, South Korea, May 2016-April 2017

A. Blood $(n=67,803)$
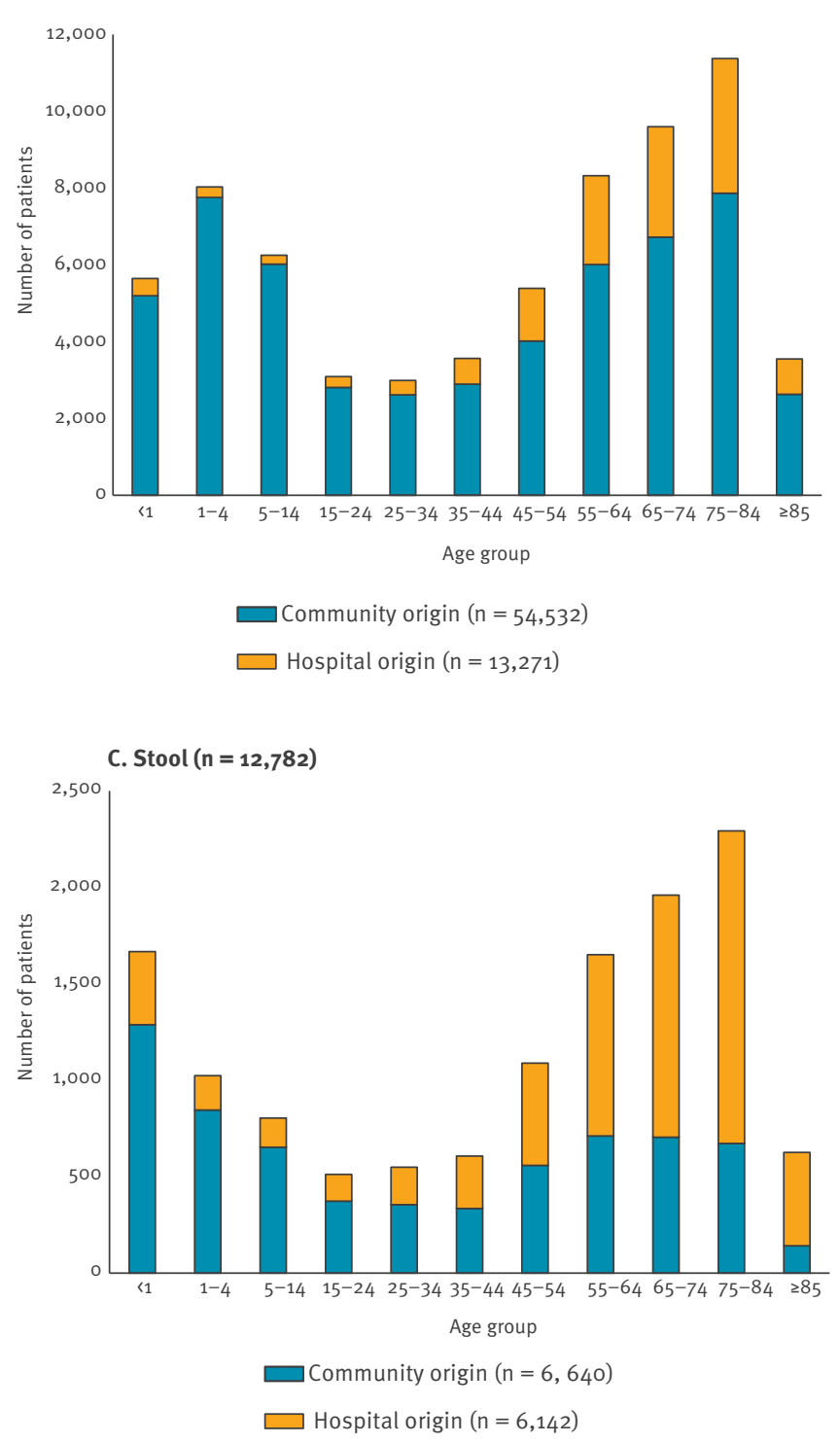

B. Urine $(n=57,477)$

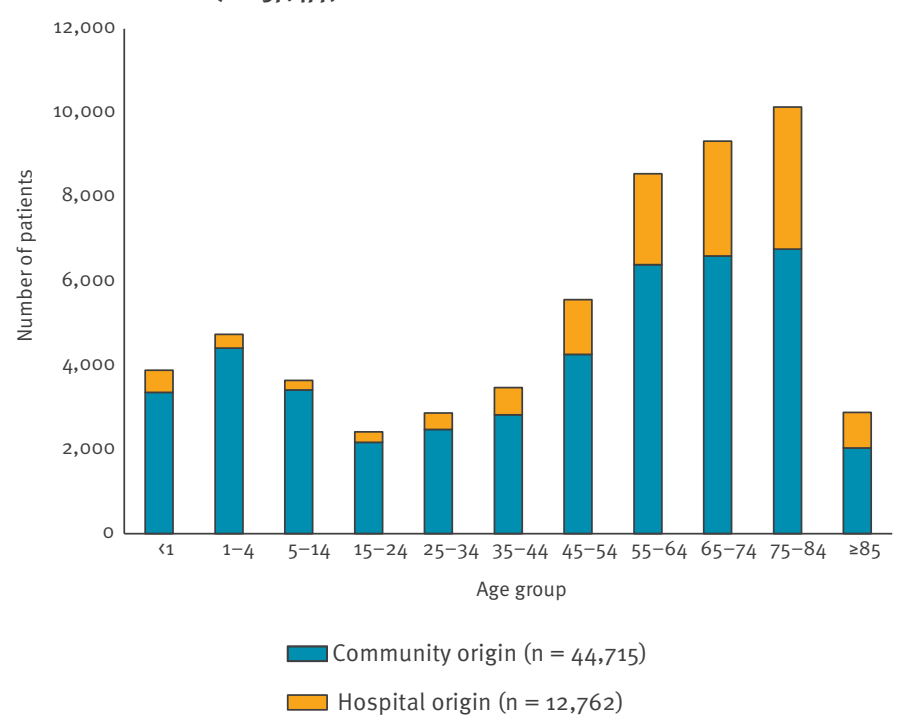

D. Genital $(n=3,111)$

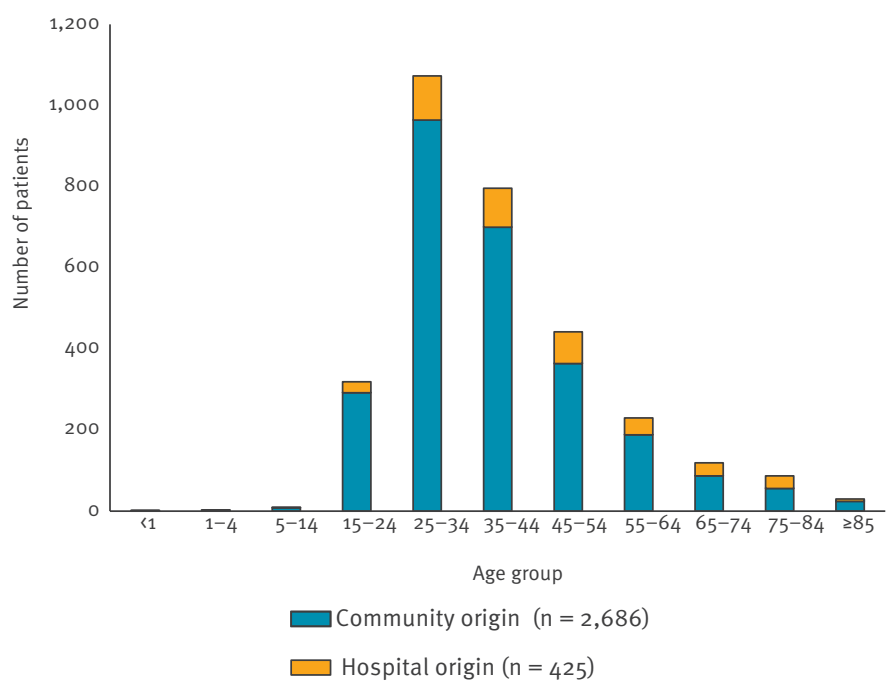

Patients are shown grouped by origin of infections. Detailed numbers can be found in Table 1 in the Supplement.

from that of GLASS: (i) three bacterial species from blood specimens were added, namely Enterococcus faecalis and Enterococcus faecium to monitor vancomycin resistance and Pseudomonas aeruginosa to monitor carbapenem resistance and (ii) more target antimicrobial agents for AST were included to investigate multidrug resistance by species.

We have operated the Kor-GLASS for one year since May 2016 and report here the first one-year assessment until April 2017.

\section{Methods}

Collection of isolates and clinical data from sentinel hospitals

The six sentinel hospitals collected bacterial isolates and clinical data, and all tests were performed in a central laboratory [5]. We collected all non-duplicated clinical isolates of Staphylococcus aureus, Streptococcus pneumoniae, Ent. faecalis, Ent. faecium, Acinetobacter spp., and $P$. aeruginosa from blood, Escherichia coli and Klebsiella pneumoniae from both blood and urine, Salmonella spp. from both blood and faeces, Shigella spp. from faeces and Neisseria 


\section{FIGURE 2}

Occurrence of bloodstream and urinary tract infections per 10,000 patient-days, by target pathogen, South Korea, May 2016-April 2017 (total patient days = 1,620,431)

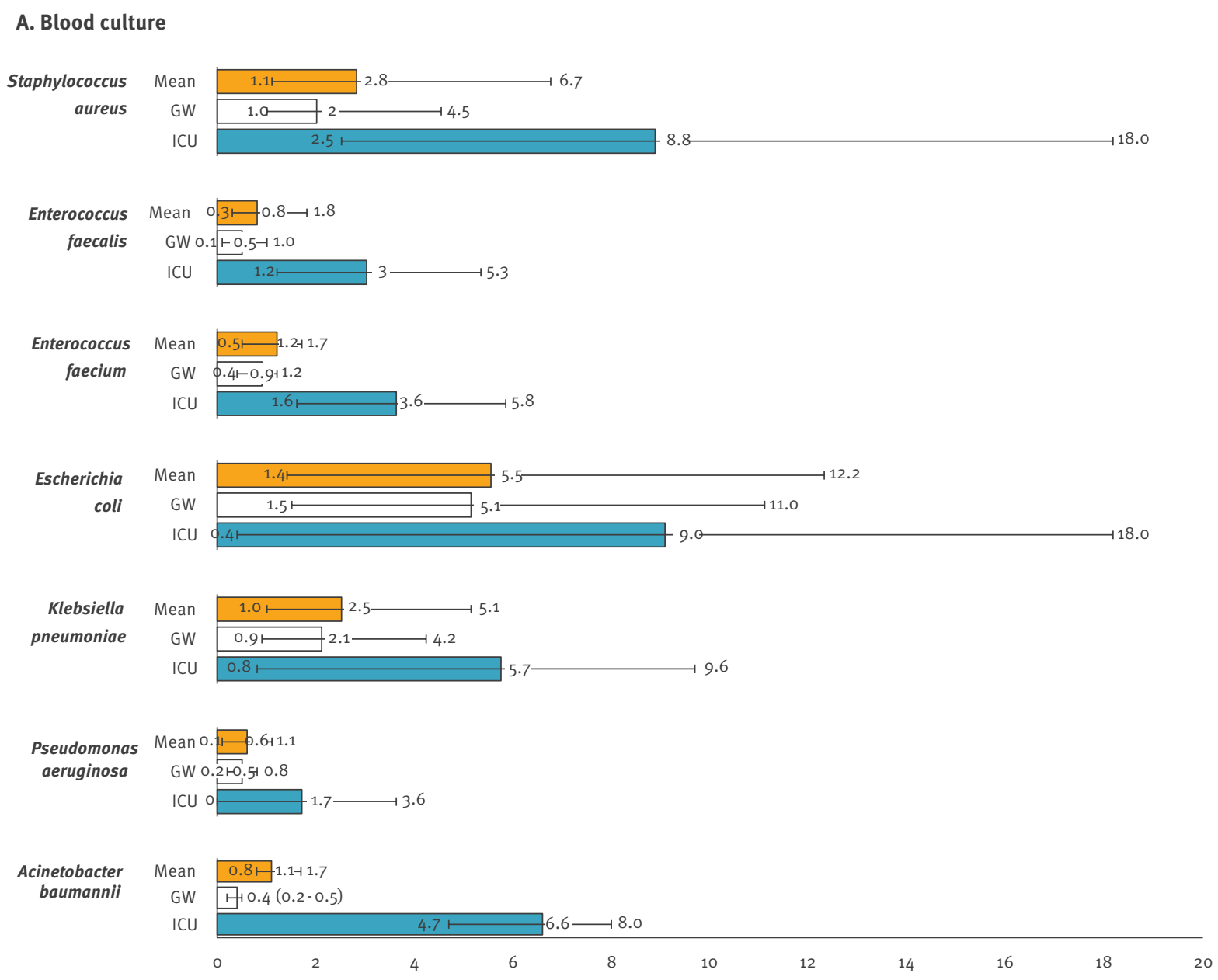

\section{B. Urine culture}

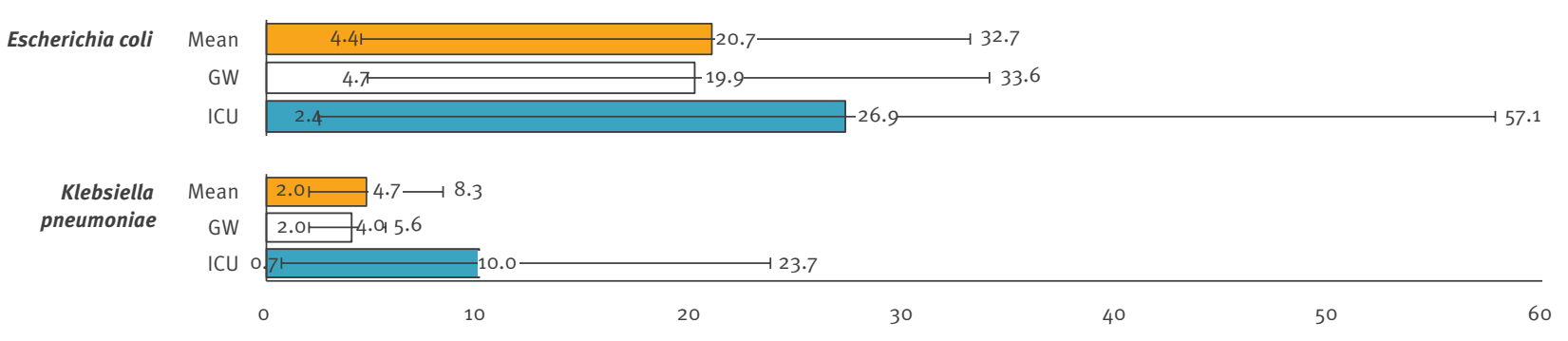

GW: general ward; ICU: intensive care unit.

The figure shows mean occurrence across all six hospitals, with the lowest and highest indicated by error bars.

gonorrhoeae from urethral and cervical swabs. Urine isolates were collected through semi-quantifying culture of urine samples following the criteria: (i) $\geq 10^{4}$ colony-forming units (CFU)/mL single-species growth of either $E$. coli or $K$. pneumoniae and (ii) $\geq 10^{5} \mathrm{CFU} / \mathrm{mL}$ of $E$. coli or K. pneumoniae in growth of mixed species [6]. We recorded epidemiological data including age, sex, infection origin (hospital origin
(HO) or community origin (CO)) and admission types (outpatient department (OPD), general ward (GW), intensive care unit (ICU)) of all patients from whom blood, urine, stool or genital cultures were taken during study period. $\mathrm{HO}$ was defined when the specimen was taken from a patient hospitalised for two or more calendar days overall, including the hospitalisation days in another healthcare facility before transfer. CO 


\section{FIGURE 3}

Percentage of resistance to major antimicrobials by infection origin, South Korea, May 2016-April 2017 (Part I: panels A-F)

\section{A. Staphylococcus aureus}

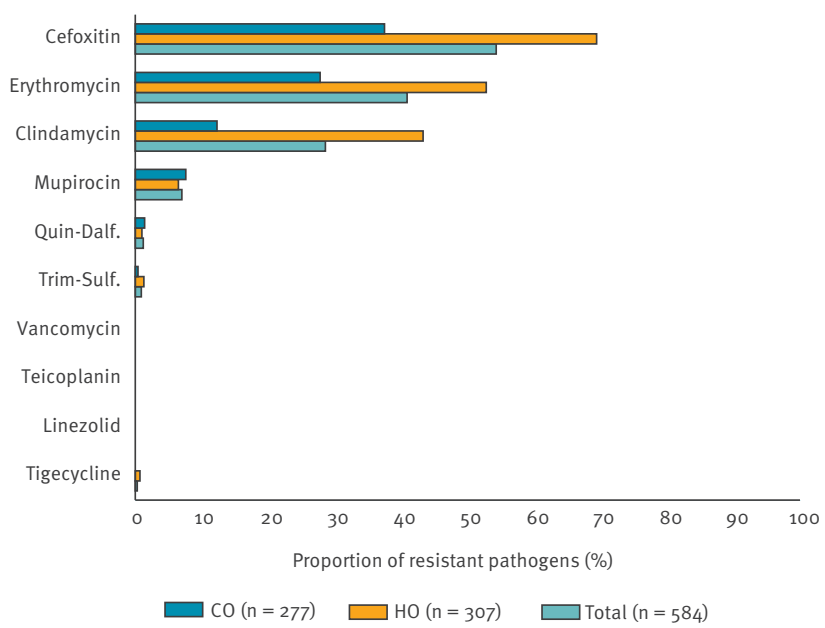

\section{Enterococus faecium}

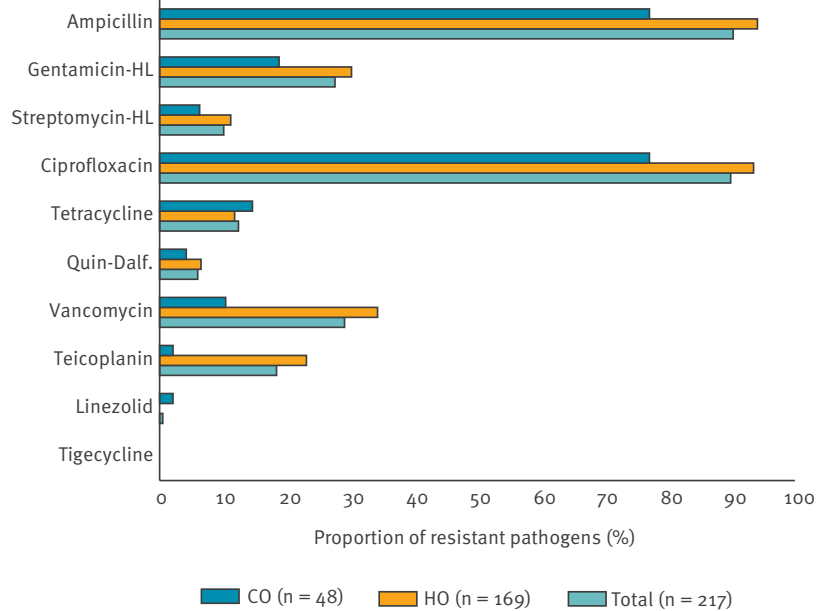

\section{E. Escherichia coli (blood)}

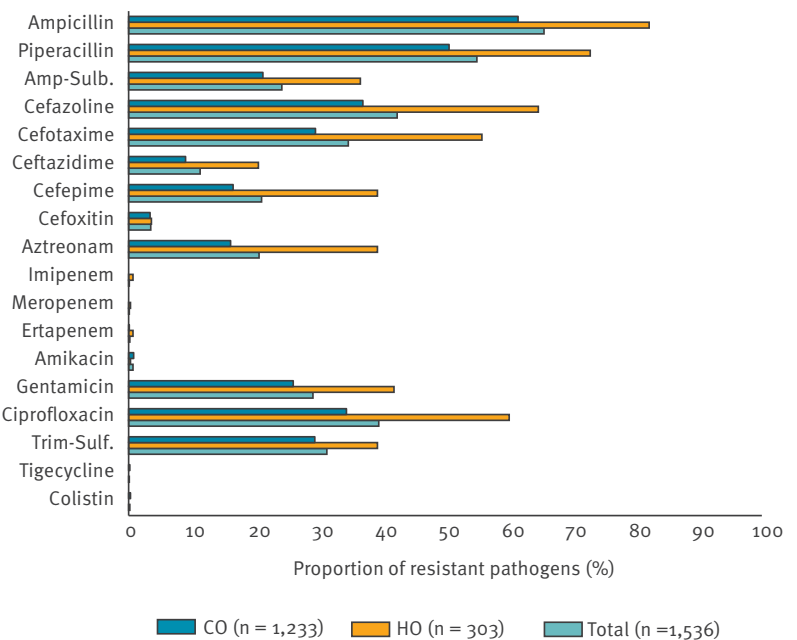

\section{B. Enterococcus faecalis}

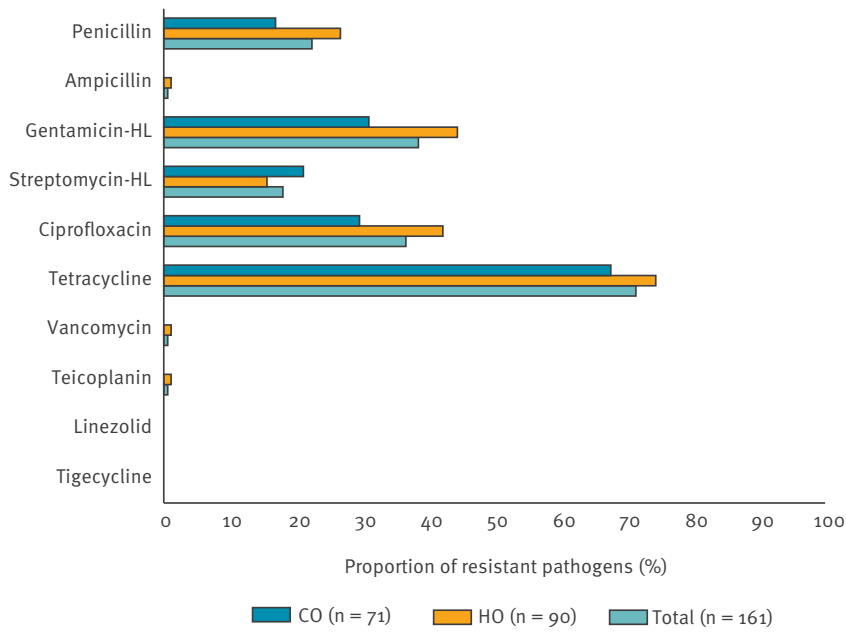

D. Streptococcus pneumoniae

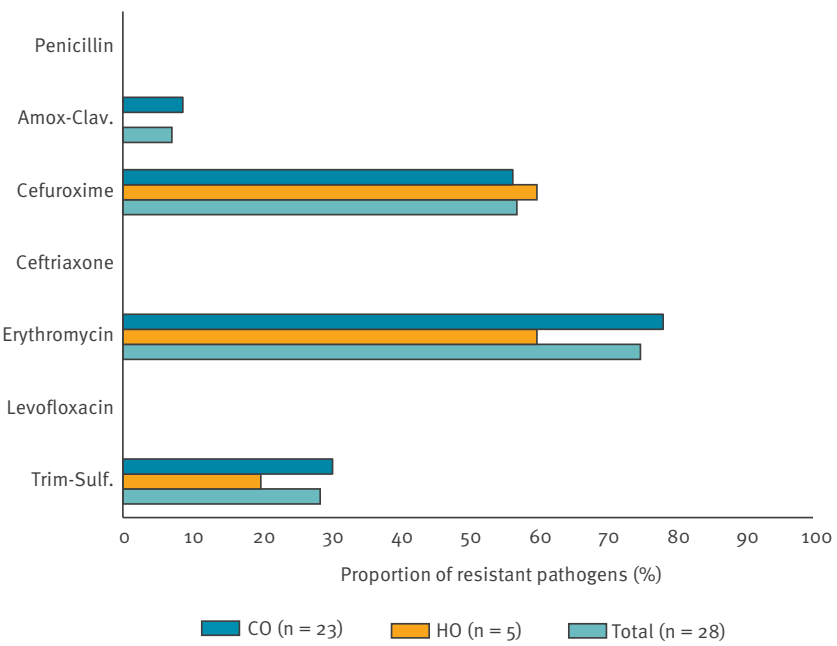

F. Escherichia coli (urine)

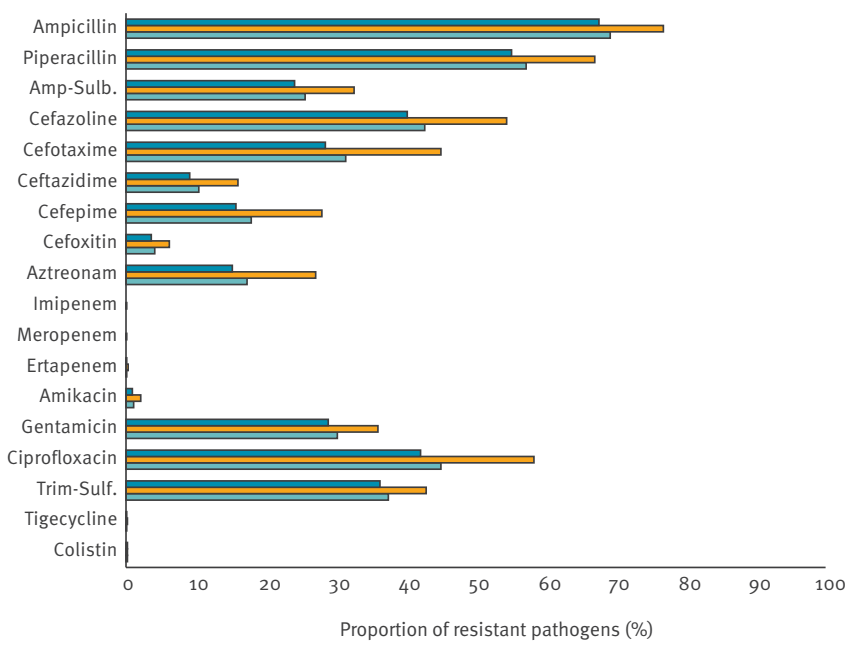

$\square$ CO (n= 5,247) $\square$ HO (n= 1,147) $\square$ Total $(n=6,394)$

Amox-Clav: amoxicillin-clavulanic acid; Amp-Sulb: ampicillin-sulbactam; CO: community origin; HO: hospital origin; Gentamicin-HL: high-level resistance to gentamicin; Pio-Tazob: piperacillin-tazobactam; Quin-Dalf: quinupristin-dalfopristin; Streptomycin-HL: high-level resistance to streptomycin; Trim-Sulf: trimethoprim-sulfamethoxazole. 


\section{FIGURE 4}

Percentage of resistance to major antimicrobials by infection origin, South Korea, May 2016-April 2017 (Part II: panels G-L)

\section{G. Klebsiella pneumoniae(blood)}

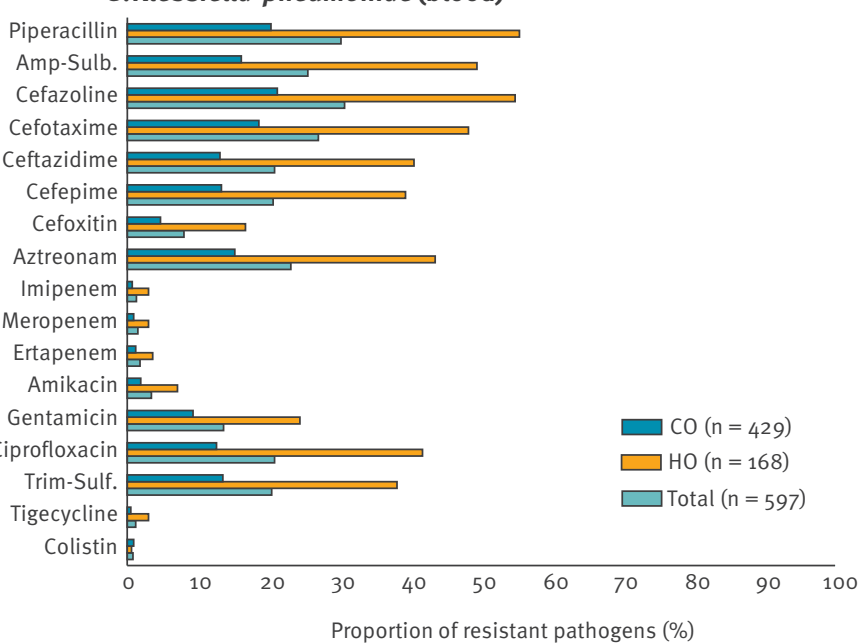

\section{Salmonella spp. (blood and faeces)}

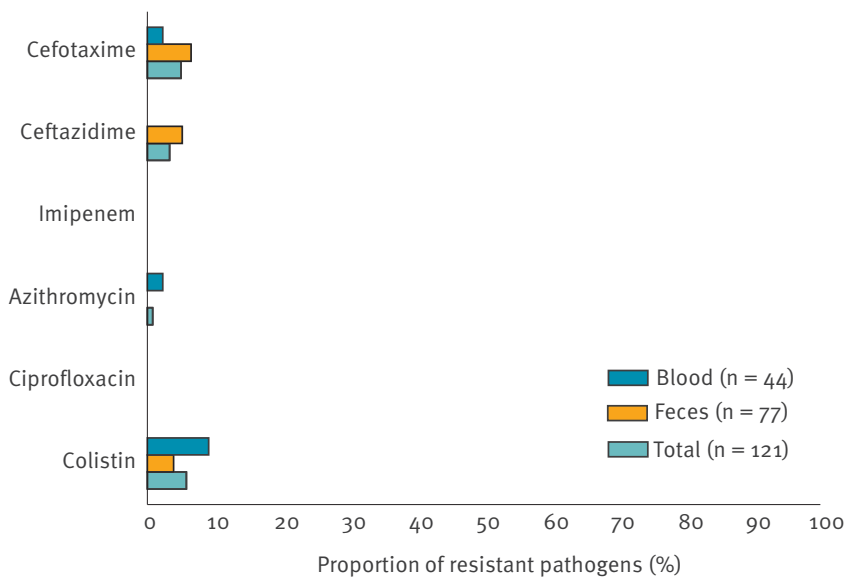

\section{K. Acinetobacter baumannii}

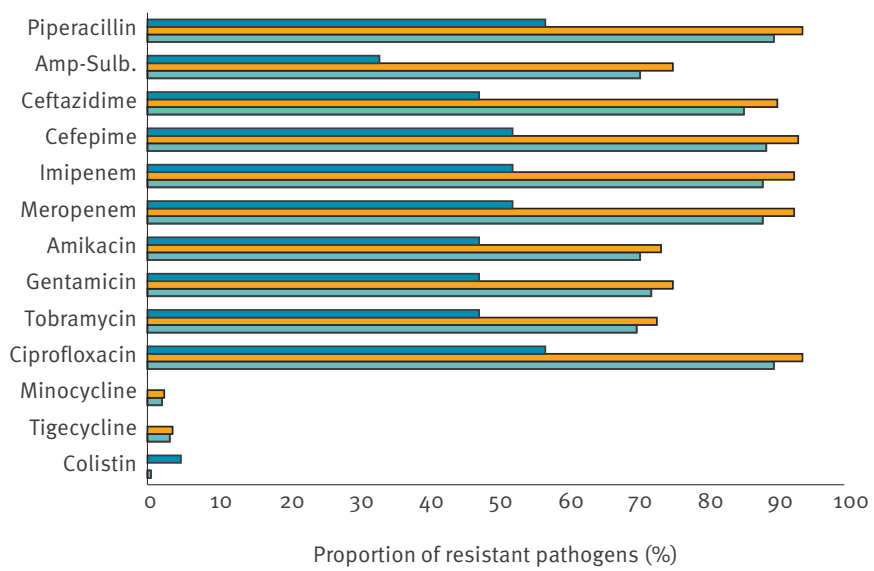

CO $(\mathrm{n}=21) \quad \square \mathrm{HO}(\mathrm{n}=167) \quad \square$ Total $(\mathrm{n}=188)$

\section{H. Klebsiella pneumoniae (urine)}

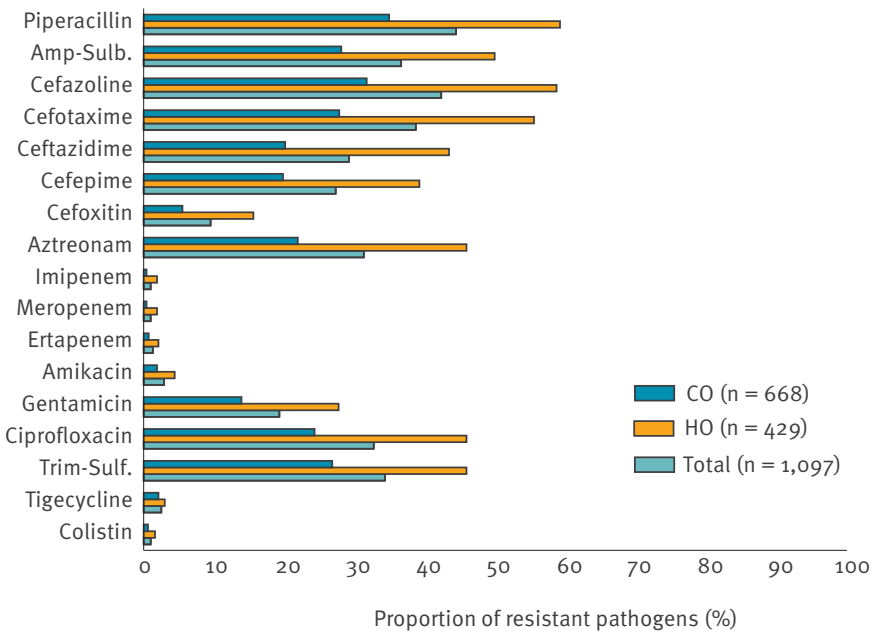

\section{J. Pseudomonas aeruginosa}

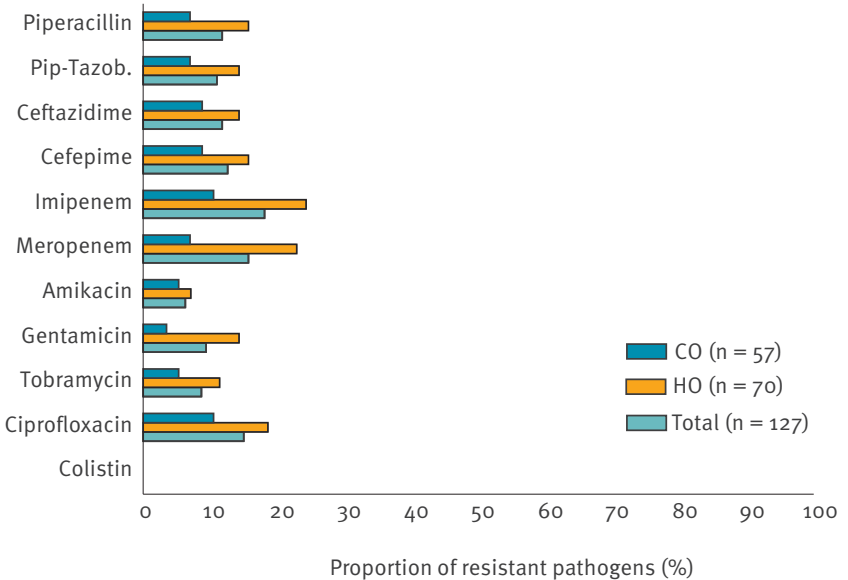

\section{Non-baumannii Acinetobacter spp.}

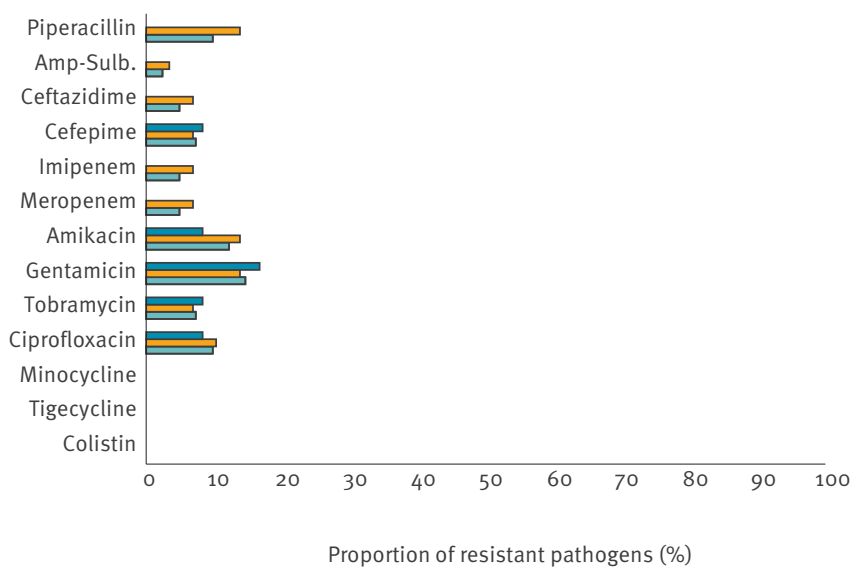

$\square \mathrm{CO}(\mathrm{n}=12) \quad \square \mathrm{HO}(\mathrm{n}=29) \quad \square$ Total $(\mathrm{n}=41)$

Amox-Clav: amoxicillin-clavulanic acid; Amp-Sulb: ampicillin-sulbactam; CO: community origin; HO: hospital origin; Gentamicin-HL: high-level resistance to gentamicin; Pio-Tazob: piperacillin-tazobactam; Quin-Dalf: quinupristin-dalfopristin; Streptomycin-HL: high-level resistance to streptomycin; Trim-Sulf: trimethoprim-sulfamethoxazole. 


\section{FIGURE 5}

Occurrence of bloodstream and urinary tract infections per 10,000 patient-days, by major antimicrobial resistant pathogen, South Korea, May 2016-April 2017 (total patient days = 1,620,431)

\section{A. Bloodstream infections}

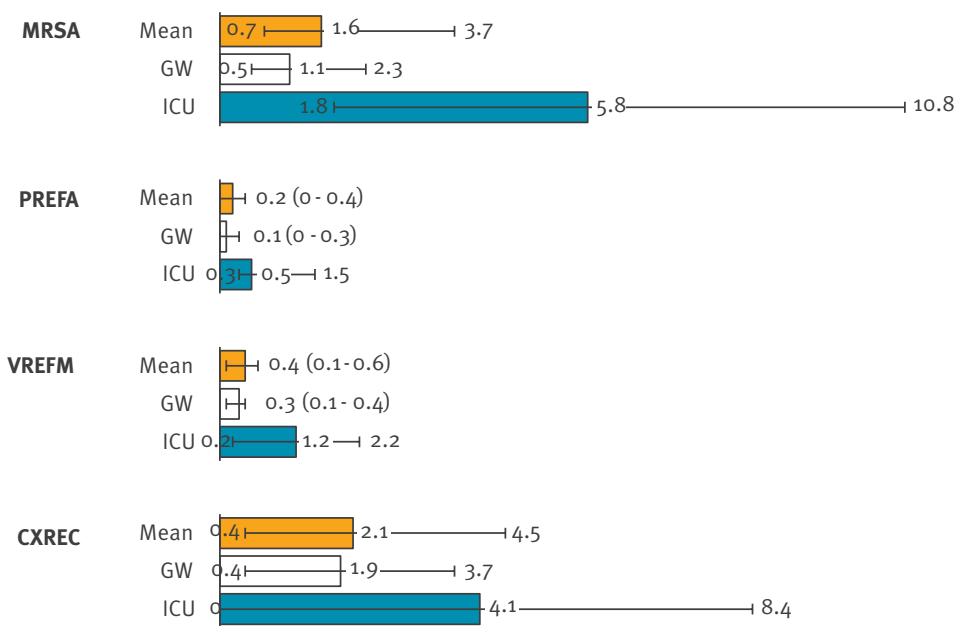

CXRKP Mean $0 \longdiv { 3 \longmapsto 0 . 8 - 1 . 7 }$

GW $\stackrel{\longrightarrow}{=} 0.6(0.3-1.1)$

$\mathrm{ICU}$

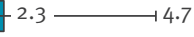

IRPA

$$
\begin{gathered}
\text { Mean } \begin{array}{l}
0.2(0-0.4) \\
\text { GW }
\end{array} \\
\text { ICU }
\end{gathered}
$$

IRAB

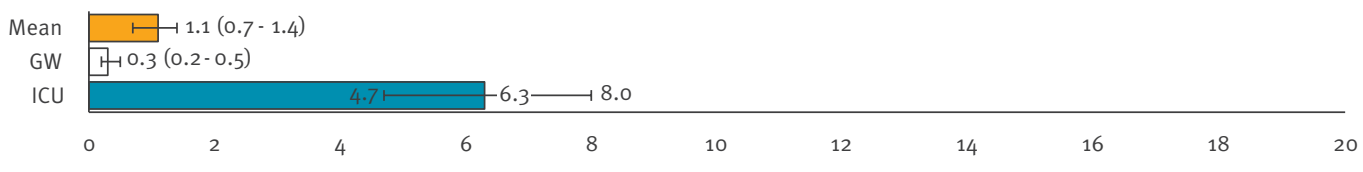

\section{B. Urinary tract infections}

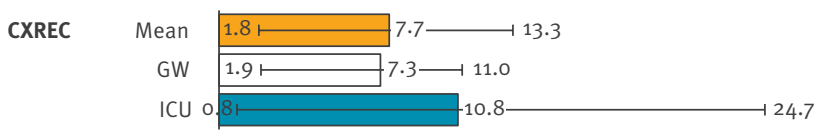

CXRKP Mean $0 . \widehat{6} 2.1-4.3$

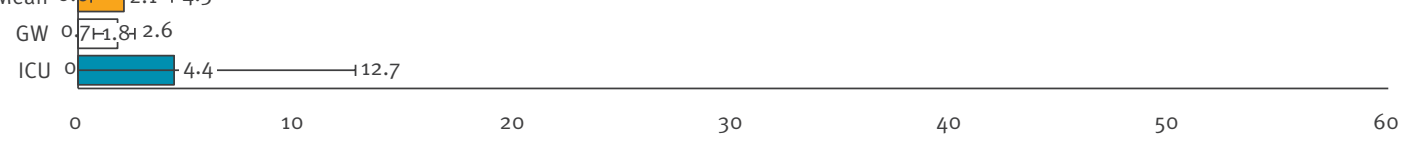

CXREC: cefotaxime-resistant Escherichia coli; CXRKP: cefotaxime-resistant Klebsiella pneumoniae; GW: General ward; ICU: intensive care unit; IRAB: imipenem-resistant Acinetobacter baumannii; IRPA: imipenem-resistant Pseudomonas aeruginosa; MRSA: meticillin-resistant Staphylococcus aureus; PREFA: penicillin-resistant Enterococcus faecalis; VREFM: vancomycin-resistant Enterococcus faecium.

The figure shows mean occurrence across all six hospitals, with the lowest and highest indicated by error bars. 
was defined when the specimen was taken either from an outpatient or from a patient hospitalised for less than 2 calendar days.

\section{Microbiological analysis in the central laboratory}

Bacterial species were re-checked in the central laboratory using a Bruker Biotyper (Bruker Daltonics $\mathrm{GmbH}$, Bremen, Germany) and/or by nucleotide sequence analysis of the $16 \mathrm{~S}$ rDNA or rpoB (for Acinetobacter sp p.). AST was carried out by disk diffusion, Etest, and broth microdilution and agar dilution methods following the Clinical and Laboratory Standards Institute guidelines [7]. Antimicrobial susceptibility phenotypes were categorised according to Magiorakos et al. [8] with a few modifications:

- fully susceptible (DS): susceptible to all tested drugs;

- drug-resistant (DR): non-susceptible to one or two drug classes;

- multidrug-resistant (MDR): non-susceptible to three or more antimicrobial classes;

- extensively drug-resistant (XDR): susceptible to two or fewer antimicrobial classes;

- pandrug-resistant (PDR): not susceptible to any antimicrobial class.

Double-blinded inter-laboratory parallel tests were conducted monthly for randomly selected isolates in order to evaluate proficiency of the results from central and the national KCDC reference laboratories.

\section{Results}

\section{Target pathogen isolation from the collected} cultures

During the 1-year period, six sentinel hospitals sampled 67,803 patients for blood culture, 57,477 for urine, 12,782 for stool and 3,111 for genital cultures (Figure 1). A total of $3,523(5.2 \%)$ target pathogens were recovered from blood (Supplement: supplementary figure 1 ). The predominant bacterial species was $E$. coli $(\mathrm{n}=1,536,43.6 \%)$, followed by $K$. pneumoniae ( $\mathrm{n}=597,16.9 \%)$ and Sta. aureus $(\mathrm{n}=584$, $16.6 \%)$. Acinetobacter spp. was recovered more often $(n=229,6.5 \%)$ than $P$. aeruginosa $(n=127,3.6 \%)$. The majority of Acinetobacter spp. were A. baumannii $(188 / 229,82.1 \%)$; the remaining 41 were nonbaumannii Acinetobacter spp. (NBA), composed of $A$. nosocomialis $(n=23)$, A. pittii $(n=12)$, A. bereziniae $(n=2), A$. soli $(n=2), A$. radioresistens $(n=1)$ and Acinetobacter genomospecies 14TU $(n=1)$. Ent. faecium ( $\mathrm{n}=217,6.2 \%$ ) was recovered more often than Ent. faecalis $(\mathrm{n}=161,4.6 \%)$. Salmonella spp. $\quad(\mathrm{n}=44$, $1.2 \%)$ and Str. pneumoniae $(\mathrm{n}=28,0.8 \%)$ were rarely recovered.
Rates of bloodstream infection (BSI) occurrence among inpatients per 10,000 patient-days (10TPD) were calculated by bacterial pathogen (Figure 2). The total number of patient-days in the six sentinel hospitals during the surveillance period was $1,620,431$ days, comprising 181,967 days in ICU and 1,438,464 days on a GW. The highest mean rate of BSI was found for $E$. coli with 5.5 (range: $1.4-12.2$ by hospital), followed by 2.8 for Sta. aureus (range: 1.1-6.7), 2.5 for $K$. pneumoniae (range: 1.0-5.1), 1.2 for Ent. faecium (range: 0.5-1.7), 1.1 for $A$. baumannii (range: $0.8-1.7$ ), 0.8 for Ent. faecalis (range: $0.3-1.8$ ) and 0.6 for $P$. aeruginosa (range: 0.1-1.1). BSI occurrence was more common in ICU patients than in GW patients: the ratio of ICU:GW was highest in A. baumannii at 16.5 (6.6:0.4), followed by 6.0 (3.0:0.5) in Ent. faecalis, 4.4 (8.8:2.0) in Sta. aureus, 4.0 (3.6:0.9) in Ent. faecium, 3.4 (1.7:0.5) in P. aeruginosa, $2.7(5.7: 2.1)$ in K. pneumoniae, and 1.8 (9.0:5.1) in E. coli.

From the 57,477 urine cultures, 6,394 (11.1\%) E. coli and 1,097 (1.9\%) K. pneumoniae were recovered. The mean rate of $E$. coli urinary tract infection (UTI) occurrence among inpatients per 10TPD was 20.7 (range: 4.4-32.7 by hospital) and that caused by $K$. pneumoniae was 4.7 (range: $2.0-8.3$ ). The ratio of UTI occurrence in ICU:GW was higher in K. pneumoniae $(2.5,10.0: 4.0)$ than in $E$. coli (1.4, 26.9:19.9). From the 12,782 stool cultures, 77 Salmonella spp. (0.6\%) were recovered. None of the 3,111 genital cultures were positive for $N$. gonorrhoeae.

\section{Antimicrobial susceptibilities of major pathogens}

\section{Gram-positive pathogens}

More than half $(317 / 584,54.3 \%)$ of Sta. aureus blood isolates were resistant to cefoxitin (Figures 3 and 4), which means that these were meticillin-resistant Sta. aureus (MRSA). By origin of infection, 69.4\% (213/307) of HO Sta. aureus were MRSA, more than the $37.5 \%$ $(104 / 277)$ of CO. Most of the isolates remained susceptible to linezolid, tigecycline and quinupristindalfopristin, and they were all susceptible to vancomycin and teicoplanin. The Sta. aureusblood isolates were categorised as DS (35.8\%, $n=209)$, DR (9.9\%, $n=58)$ and MDR $(54.3 \%, n=317)$, and all MDR isolates were MRSA (Supplement: supplementary figure 2).

While resistance to ampicillin in Ent. faecalis blood isolates was rare $(1 / 161,0.6 \%)$, the proportion of penicillin resistance was comparably high $(n=36$, $22.4 \%)$ and more frequent in $\mathrm{HO}(24 / 90,26.7 \%)$ than in $\mathrm{CO}(12 / 71,16.9 \%)$. Resistance to glycopeptides was also rare (Figures 3 and 4 ). The rate of high-level resistance [7] to aminoglycosides was 38.5\% $(n=62)$ to gentamicin and $18.0 \%(n=29)$ to streptomycin. The majority of the Ent. faecalis blood isolates were either DR $(49.1 \%, n=79)$ or $\operatorname{MDR}(42.2 \%, n=68)$. The proportion of multidrug resistance in penicillinresistant isolates was three times higher $(86.1 \%$, $31 / 36)$ than that in susceptible ones $(29.6 \%, 37 / 125)$. 
Among the 217 Ent. faecium blood isolates, 90.3\% $(n=196)$ were resistant to ampicillin and $29.0 \%$ $(n=63)$ and $18.4 \% \quad(n=40)$ were resistant to vancomycin and teicoplanin, respectively. For these drugs, resistance rates in $\mathrm{HO}$ isolates were higher than those in $\mathrm{CO}$ isolates (Figures 3 and 4). Forty of the 63 vancomycin-resistant Ent. faecium (VREFM) were also resistant to teicoplanin and 14 were intermediate, while nine remained susceptible to the drug. In addition, $27.6 \%(\mathrm{n}=60)$ and $10.1 \%(\mathrm{n}=22)$ of all Ent. faecium were high-level resistant to gentamicin and streptomycin, respectively. Two thirds of the Ent. faecium blood isolates were MDR $(62.7 \%, \mathrm{n}=136)$ and one third was DR $(33.2 \%, n=72)$. All VREFM isolates were categorised as MDR.

All Str. pneumoniae blood isolates were susceptible $(23 / 28)$ or intermediate $(n=5)$ to penicillin. The five penicillin-intermediate isolates were also nonsusceptible to other drugs. All 28 isolates were susceptible to levofloxacin. Erythromycin resistance was observed in 21 of the 28 isolates.

\section{Enterobacteriaceae}

Ampicillin resistance was identified in $65.6 \%$ $(1,007 / 1,536)$ of $E$. coli blood isolates and decreased to $24.2 \% \quad(n=372)$ when sulbactam was supplemented. Rates of resistance to cefotaxime, ceftazidime and cefepime were $34.7 \%(n=533), 11.3 \%(n=173)$ and $21.0 \%(n=322)$, respectively. Carbapenem-nonsusceptible isolates were rarely identified. The resistance rate to ciprofloxacin was $39.5 \%(n=606)$ and that to amikacin was low at $0.7 \%(n=11)$. Colistinresistant isolates were seldom identified, with $0.2 \%$ $(n=3)$. Resistance rates in $E$. coli urine isolates were similar to those in blood isolates. One isolate was resistant to all three carbapenems and six were nonsusceptible only to ertapenem. Colistin resistance was detected in $14(0.2 \%)$ of the $6,394 \mathrm{E}$. coli urine isolates. $\mathrm{HO}$ isolates had higher resistance rates to most of the tested antimicrobials than CO isolates, and this difference was much greater in blood isolates than in urine isolates. The vast majority $(98.8 \%, 2,616 / 2,648)$ of cefotaxime-non-susceptible $E$. coli isolates were MDR and all XDR isolates $(0.2 \%, 15 / 7,930)$ were nonsusceptible to both carbapenems and cefotaxime.

One third $(30.2 \%, 180 / 597)$ of $K$. pneumoniae blood isolates were piperacillin-resistant and a quarter $(25.5 \%, n=152)$ were resistant to ampicillin-sulbactam. For the extended-spectrum cephalosporins, $27.0 \%$ $(n=161)$ isolates were resistant to cefotaxime, $20.8 \%$ $(\mathrm{n}=124)$ to ceftazidime and $20.6 \%(\mathrm{n}=123)$ to cefepime. Carbapenem resistance was identified more frequently in $K$. pneumoniae blood isolates than in $E$. coli (Figures 3 and 4 ). The resistance rate to ciprofloxacin was $20.8 \%(n=124)$, and those to amikacin and gentamicin were $3.4 \%(n=20)$ and $13.6 \%(n=81)$, respectively. Colistin resistance was identified in $0.8 \%$ $(n=5)$ isolates. K. pneumoniae urine isolates exhibited higher rates of resistance to the antimicrobials tested than the blood isolates. Carbapenem resistance rates in the 1,097 urine isolates were $1.0 \%(n=11)$ to imipenem, $1.0 \%(n=11)$ to meropenem, and $1.3 \%(n=14)$ to ertapenem. Colistin resistance was detected in $1.0 \%(n=11)$ isolates. The proportion of antimicrobial resistance in $K$. pneumoniae of $\mathrm{HO}$ was higher than in those of $\mathrm{CO}$ and the difference between them was larger in blood isolates (ca. three times higher) than in urine isolates (ca. 1.5 times higher). Most of the 604 Cefotaxime-non-susceptible isolates were MDR (77.6\%, $n=469)$ or $\operatorname{XDR}(20.9 \%, n=126)$.

Among 44 Salmonella blood isolates, one isolate was resistant to cefotaxime and intermediate to ceftazidime. All the isolates remained susceptible to imipenem. None was resistant to ciprofloxacin, however, 10 were intermediate to the drug, and colistin resistance was observed in four. Five of the 77 Salmonella stool isolates were cefotaxime-resistant, and four of those five were also resistant to ceftazidime. Similar to the blood isolates, all stool isolates were also susceptible to imipenem and 12 isolates were intermediate to ciprofloxacin. Three isolates were resistant to colistin.

Glucose non-fermenting Gram-negative bacilli Non-susceptibility to piperacillin in $P$. aeruginosa blood isolates was observed in 21 of 127 (16.5\%) isolates, and supplementing tazobactam had no effect on the susceptibility proportion $(82.7 \%, \mathrm{n}=105)$ (Figures 3 and 4 ). The proportion of resistance to ceftazidime and cefepime was $11.8 \%(n=15)$ and $12.6 \%(n=16)$, respectively. Rates of carbapenem resistance were $18.1 \%(n=23)$ for imipenem and $15.7 \%(n=20)$ for meropenem. In addition, $6.3 \%(n=8)$ of isolates were resistant to amikacin, $9.4 \%(n=12)$ to gentamicin, $8.7 \%(n=11)$ to tobramycin and $15.0 \%(n=19)$ to ciprofloxacin. All isolates remained susceptible to colistin. Twice as many $\mathrm{HO}$ than $\mathrm{CO}$ isolates were non-susceptible to the drug. The amikacin-non-susceptible $P$. aeruginosa blood isolates were mostly XDR (8/9) with the exception of one MDR isolate. In contrast, 14 of 30 among the imipenem-non-susceptible isolates were XDR, four were MDR and 12 were DR.

The proportion of AMR in the 188 A. baumannii blood isolates was higher than $70 \%$ for all tested drugs (Figures 3 and 4 ) and the AMR proportion in $\mathrm{HO}$ isolates was ca. twice that of $\mathrm{CO}$ isolates. Minocycline, tigecycline and colistin were still active in $93.1 \%$ $(n=175), 85.6 \%(n=161)$ and $99.5 \%(n=187)$ of isolates, respectively. Among the $A$. baumanniiblood isolates, $76.1 \%(n=143)$ and $13.8 \%(n=26)$ were XDR and MDR, respectively, and all but two MDR isolates were imipenem-non-susceptible. NBA blood isolates had a markedly lower proportion of AMR than A. baumannii.

\section{The estimated prevalence of major AMR pathogens}

In general, the AMR proportion of major pathogens for ICU patients was higher than for GW and OPD patients (Supplement: supplementary figure 3). BSI occurrence 
by major AMR pathogen in inpatients per 10TPD (Figure 5) had the highest mean value of 2.1 for cefotaximeresistant $E$. coli (CXREC), followed by 1.6 for MRSA, 1.1 for imipenem-resistant $A$. baumannii, 0.8 for cefotaxime-resistant $K$. pneumoniae (CXRKP), 0.4 for VREFM, 0.2 for penicillin-resistant Ent. faecalis (PREFA) and 0.2 for imipenem-resistant $P$. aeruginosa. As shown in Figure 5 , the ratio of $\mathrm{BSI}$ occurrence of the major AMR pathogens was much higher in ICU than on GW. Mean UTI occurrence in inpatients per 10TPD was higher for CXREC at 7.7 than for CXRKP at 2.1. The ratio of UTI occurrence in ICU vs GW was higher for CXRKP at 2.4 than for CXREC at 1.5 .

\section{Discussion}

De Kraker et al. [9] described an increasing trend in the overall incidence of bacteremia caused by the top five pathogens (E. coli, Sta. aureus, Str. pneumoniae, Ent. faecalis and Ent. faecium) between 2002 and 2008, based on the European Antimicrobial Resistance Surveillance Network (EARS-Net; https://ecdc. europa.eu/en/about-us/networks/disease-networksand-laboratory-networks/ears-net-about) database. Predominance of $E$. coli among pathogens causing BSIs is a common phenomenon worldwide. We assessed the relative incidence of BSIs caused by $E$. coli compared with other species: The relative ratio of $E$. coli to Sta. aureus in Kor-GLASS was moderate at 2.6, which was lower than the 3.2 observed in Norway [10], similar to the ratios of 2.4 in Taiwan [11] and 2.2 in Netherlands [12], and higher than the ratios of 1.2 in Japan [13], 1.2 in Vietnam [14], 1.0 in Greece [15] and 1.1 in Malawi [16]. The relative ratio of $E$. coli to $K$. pneumoniae in South Korea was also moderate at 2.6, lower than in Northern European countries ( 5.4 in the Netherlands and 5.0 in Norway), similar to 2.5 in Japan and 3.0 in Taiwan, and higher than 1.0 in Vietnam. Str. pneumoniae was frequently isolated from blood in European countries (relative ratio of $E$. coli to Str. pneumoniae: 3.2 in the Netherlands, 3.1 in Norway and 4.1 in Spain), but rarely identified in South Korea (ratio: 54.9 ) which was similar to Asian countries (ratio: 18.3 in Vietnam and 31.8 in Taiwan). The Salmonella spp. is still a major pathogen causing BSI in developing countries such as Vietnam (relative ratio of $E$. coli to Salmonella spp.: 1.4) and Malawi (ratio: 0.2), however, this species was seldom identified in South Korea (ratio: 34.9), similar to the ratios 28.1 in Spain and 14.3 in Taiwan.

Sta. aureus was the second most common pathogen causing BSI following $E$. coli. BSI caused by Sta. aureus occurred in 2.8 inpatients per 10TPD in our study, which is 1.8 -fold more than the 1.6 inpatients per 10TPD measured in 2011 in a previous prospective multi-center study in South Korea [17]. Since the surveillance system of that study is not compatible to Kor-GLASS, the potential increase in Sta. aureus-BSI needs to be further followed up. Similarly, we observed an increased incidence of MRSA-BSI at 1.6 inpatients per 10TPD compared with 1.2 inpatients per 10TPD in 2011. This incidence was 2.5 times higher than the 0.62 inpatients per 10TBD reported in a Canadian surveillance study in 2014 [18]. The high prevalence of MRSA in blood isolates in South Korea has decreased from $72 \%$ in 2013 to $66 \%$ in 2015 as measured by KARMS [4] and further to $54.3 \%$ in 2016 according to Kor-GLASS data. EARS-Net reported similarly that the percentage of MRSA has decreased from $18.1 \%$ in 2013 to $13.7 \%$ in 2016 [9]. In addition, MRSA occurred more frequently in ICU patients than in GW patients ( 5.8 vs 1.1), indicating that MRSA is a problem in ICUs.

Enterococci have become a major cause of BSIs globally, owing to their intrinsic resistance to various antibiotics and their enormous ability to acquire resistance to antimicrobials. Ent. faecium and Ent. faecalis were the fourth and sixth most common pathogens causing $\mathrm{BSI}$ in inpatients in our study, with 1.2 and $0.8 \mathrm{BSI}$ occurrences per 10TPD, respectively, and they caused over four times more BSI in ICUs than on GWs. Ent. faecium exhibited remarkably higher resistance rates than Ent. faecalis to ampicillin (90.3\% vs 0.6\%) and vancomycin (29.1\% vs $0.6 \%$ ), resulting in the identification of 1.3 -fold more Ent. faecium than Ent. faecalis, similar to the 1.8-fold difference found in Spain [19]. A reversed ratio was observed in the Netherlands (0.8:1) [14], and Japan (0.7:1) [12], countries that have low rates of AMR to these drugs. The vancomycin resistance rate (29.1\%) in Ent. faecium was higher than that of teicoplanin (18.4\%) in South Korea, although all isolates were vanA-positive. This might be caused by the dissemination of clones with a vanA genotypeVanA phenotype along with a vanA genotype-VanD phenotype following inactivation of vanYand vanZ in the vanA operon by rearrangement of Tn1546 [20]. PREFA was also a common BSI-causative AMR pathogen, especially in ICUs. Clinical impacts of the penicillin resistance need to be further investigated.

Both $E$. coli and $K$. pneumoniae exhibited higher resistance rates to cefotaxime than to ceftazidime owing to the dissemination of CTX-M-type extended-spectrum $\beta$-lactamases (ESBLs). The difference in the resistance rates to these drugs was greater in $E$. coli than in $K$. pneumoniae, which could have two possible causes: higher prevalence $K$. pneumoniae compared with $E$. coli (i) of CTX-M group 1 ESBLs, which have an expanded hydrolytic activity to ceftazidime, and (ii) of SHV-type ESBLs, which hydrolyse both cefotaxime and ceftazidime [21,22]. Identification of carbapenemase-producing Enterobacteriaceae (CPEs) in our surveillance study seemed the tip of an iceberg of the notorious AMR pathogen disseminated in clinical settings. All CPEs we identified were $K$. pneumoniae carbapenemase (KPC) producers. Continuous monitoring and action plans for CPEs are required because various types of carbapenemases, including KPC, New Delhi metallo$\beta$-lactamase and OXA-48-likes, have been introduced to South Korea during the last decade [23-25]. Colistin resistance in Enterobacteriaceae was rare in our study and none contained the mobile colistin resistance (mcr) genes $m c r-1$ to $m c r-5$ [26-28], although clinical 
Enterobactericeae isolates carrying the $\mathrm{mcr}$-1 gene have already been reported in South Korea [29].

Amikacin non-susceptibility was a better indicator for XDR $P$. aeruginosa than imipenem-non-susceptibility. The carbapenemase-producing $P$. aeruginosa strains in South Korea harboured a class 1 integron carrying several resistance gene cassettes coding not only for a carbapenemase, but also for aminoglycoside-modifying enzymes [30]. Thus, most of the amikacin-nonsusceptible isolates were XDR. However, carbapenem resistance in $P$. aeruginosastrains was conferred not only through acquisition of a gene for carbapenemase, but also through loss or alteration of the intrinsic OprD porin or overproduction of the efflux pumps [31], which rarely confer resistance to amikacin to the bacterial hosts.

BSIs caused by $A$. baumannii strains mostly occurred in ICUs rather than on GWs [32]. The likely reason is that the $A$. baumannii BSIs originated predominantly from pulmonary infections associated with ventilators. The rate of carbapenem resistance in $A$. baumannii strains was markedly high at $89.9 \%$ and most of these strains were XDR. This result is in line with previous reports from South Korea, and the resistance was mostly associated with OXA-23 carbapenemase production [33].

\section{Conclusion}

The Kor-GLASS, which performs strain collection and centralised analysis, was launched and operated. It provided well-curated surveillance data devoid of collection bias or isolate duplication, including patient data associated with the bacterial cultures. In addition, frequency of infection occurrence by patient-days was estimated for comparison with foreign countries. The results of this surveillance helped plan national action in response to the high rate of drug resistance. A bacterial bank and a database for the collections are under development.

\section{Acknowledgements}

Ethical statement: The research, which has no involvement of human subjects but the clinical isolates, does meet the exempt category without approval from Ethics Committee on Human Research of the Health Ministry in South Korea and the study design has not been reviewed by the committee.

Funding: this work was supported by the Research Programme funded by the Korean Centers for Disease Control and Prevention (2016ER230100\#).

\section{Conflict of interest}

None to declare.
Authors' contributions

SHJ, KJL: conceiving the surveillance project; SHJ, JWY, IHK, $\mathrm{CP}, \mathrm{KJL}$ : managing the study to run properly; HL, E-JY, DK, SHJ, KJL: operating the surveillance system; HL, E-JY, DK, SHJ: analysing the data; HL, E-JY, SHJ: writing the manuscript; EJW, JHS3, SHK, JHS 5 , KSS, YAK, YU: responsible for collecting the bacterial strains and clinical data; JHS3, JHS5, KSS: critically reading the manuscript.

\section{References}

1. World Health Organization (WHO). Antimicrobial resistance: global report on surveillance 2014. Geneva: WHO; 2014. Available from: http://www.who.int/drugresistance/ documents/surveillancereport/en/

2. World Health Organization (WHO). Global antimicrobial resistance surveillance system: Manual for early implementation. Geneva: WHO; 2015. Available from: http://www.who.int/antimicrobial-resistance/publications/ surveillance-system-manual/en/

3. Hong SG, Yong D, Lee K, Kim EC, Lee WK, Jeong SH, et al. Antimicrobial resistance of clinically important bacteria isolated from hospitals located in representative provinces of Korea. Korean J Clin Microbiol. 2003;6(1):29-36. Korean.

4. Kim D, Ahn JY, Lee CH, Jang SJ, Lee H, Yong D, et al. Increasing resistance to extended-spectrum cephalosporins, fluoroquinolone, and carbapenem in Gram-negative bacilli and the emergence of carbapenem non-susceptibility in Klebsiella pneumoniae: Analysis of Korean Antimicrobial Resistance Monitoring System (KARMS) data from 2013 to 2015. Ann Lab Med. 2017;37(3):231-9. https://doi.org/10.3343/ alm.2017.37.3.231 PMID: 28224769

5. Lee H, Yoon EJ, Kim D, Jeong SH, Shin JH, Shin JH, et al. Establishment of the South Korean national antimicrobial resistance surveillance system, , Kor-GLASS, in 2016. Euro Surveill. 2018;23(42):1700734.

6. Clinical microbiology procedures handbook. 4th ed. Leber AL, editor. Washington D.C.: ASM Press; 2016.

7. Clinical and Laboratory Standards Institute (CLSI). Performance standards for antimicrobial susceptibility testing. CLSI Document M10oS. 26th ed. Wayne: CLSI; 2016. Available from: http://lizx.cqrmhospital.com/ upfiles/201601/20160112155335884.pdf

8. Magiorakos AP, Srinivasan A, Carey RB, Carmeli Y, Falagas ME, Giske CG, et al. Multidrug-resistant, extensively drugresistant and pandrug-resistant bacteria: an international expert proposal for interim standard definitions for acquired resistance. Clin Microbiol Infect. 2012;18(3):268-81. https:// doi.org/10.1111/j.1469-0691.2011.03570.x PMID: 21793988

9. de Kraker ME, Jarlier V, Monen JC, Heuer OE, van de Sande N, Grundmann H. The changing epidemiology of bacteraemias in Europe: trends from the European Antimicrobial Resistance Surveillance System. Clin Microbiol Infect. 2013;19(9):860-8. https://doi.org/10.1111/1469-0691.12028 PMID: 23039210

10. Mehl A, Åsvold BO, Lydersen S, Paulsen J, Solligård E, Damås JK, et al. Burden of bloodstream infection in an area of Mid-Norway 2002-2013: a prospective population-based observational study. BMC Infect Dis. 2017;17(1):205-18. https://doi.org/10.1186/s12879-017-2291-2 PMID: 28284196

11. Hsieh WS, Tsai YT, Chi WM, Wu HH. Epidemiology and prevalence of bloodstream infections in a regional hospital in Northern Taiwan during 2008-2013. J Exp Clin Med. 2014;6(6):187-9. https://doi.org/10.1016/j.jecm.2014.10.011

12. Altorf-van der Kuil W, Schoffelen AF, de Greeff SC, Thijsen SF, Alblas HJ, Notermans DW, et al. The National Amr Surveillance Study Group. National laboratory-based surveillance system for antimicrobial resistance: a successful tool to support the control of antimicrobial resistance in the Netherlands. Euro Surveill. 2017;22(46):00062. https://doi.org/10.2807/15607917.ES.2017.22.46.17-00062 PMID: 29162208

13. Japan Nosocomial Infectious Surveillance (JANIS). Annual open report 2015 (all facilities). Tokyo: JANIS; 2017. Available from: https://janis.mhlw.go.jp/english/report/open report/2015/4/1/ken_Open_Report_Eng_201500_clsi2012.pdf

14. Dat VQ, Vu HN, Nguyen The H, Nguyen HT, Hoang LB, Vu Tien Viet $D$, et al. Bacterial bloodstream infections in a tertiary infectious diseases hospital in Northern Vietnam: aetiology, drug resistance, and treatment outcome. BMC Infect Dis. 2017;17(1):493-503. https://doi.org/10.1186/s12879-017-25827 PMID: 28701159

15. Kolonitsiou F, Papadimitriou-Olivgeris $M$, Spiliopoulou A, Stamouli V, Papakostas V, Apostolopoulou E, et al. Trends of bloodstream infections in a university Greek 
hospital during a three-year period: Incidence of multidrugresistant bacteria and seasonality in Gram-negative predominance. Pol J Microbiol. 2017;66(2):171-80. https://doi. org/10.5604/01.3001.0010.7834 PMID: 28735318

16. Musicha P, Cornick JE, Bar-Zeev N, French N, Masesa C, Denis $B$, et al. Trends in antimicrobial resistance in bloodstream infection isolates at a large urban hospital in Malawi (19982016): a surveillance study. Lancet Infect Dis. 2017;17(10):104252. https://doi.org/10.1016/S1473-3099(17)30394-8 PMID: 28818544

17. Kim CJ, Kim HB, Oh MD, Kim Y, Kim A, Oh SH, et al. KIND Study group (Korea Infectious Diseases Study group). The burden of nosocomial staphylococcus aureus bloodstream infection in South Korea: a prospective hospital-based nationwide study. BMC Infect Dis. 2014;14(1):590. https://doi.org/10.1186/ S12879-014-0590-4 PMID: 25891200

18. Public Health Agency of Canada (PHAC). 2016. Canadian antimicrobial resistance surveillance system - Report 2016. Ottawa: PHAC; 2016. Available from: https://www.canada.ca/ content/dam/phac-aspc/documents/services/publications/ drugs-health-products/antibiotic-resistance-antibiotique/ antibiotic-resistance-antibiotique-2016-eng.pdf

19. Royo-Cebrecos C, Gudiol C, Ardanuy C, Pomares H, Calvo M, Carratalà J. A fresh look at polymicrobial bloodstream infection in cancer patients. PLoS One. 2017;12(10):e0185768. https:// doi.org/10.1371/journal.pone.0185768 PMID: 29065118

20. Lee WG, Huh JY, Cho SR, Lim YA. Reduction in glycopeptide resistance in vancomycin-resistant enterococci as a result of vanA cluster rearrangements. Antimicrob Agents Chemother. 2004;48(4):1379-81. https://doi.org/10.1128/AAC.48.4.13791381.2004 PMID: 15047548

21. Kim MH, Lee HJ, Park KS, Suh IT. Molecular characteristics of extended spectrum beta-lactamases in Escherichia coli and Klebsiella pneumoniae and the prevalence of qnr in Extended spectrum beta-lactamase isolates in a tertiary care hospital in Korea. Yonsei Med J. 2010;51(5):768-74. https://doi. org/10.3349/ymj.2010.51.5.768 PMID: 20635454

22. Poirel L, Gniadkowski M, Nordmann P. Biochemical analysis of the ceftazidime-hydrolysing extendedspectrum beta-lactamase CTX-M-15 and of its structurally related beta-lactamase CTX-M-3. J Antimicrob Chemother. 2002;50(6):1031-4. https://doi.org/10.1093/jac/dkf240 PMID: 12461028

23. Kim MN, Yong D, An D, Chung HS, Woo JH, Lee K, et al. Nosocomial clustering of NDM-1-producing Klebsiella pneumoniae sequence type 340 strains in four patients at a South Korean tertiary care hospital. J Clin Microbiol. 2012;50(4):1433-6. https://doi.org/10.1128/JCM.06855-11 PMID: 22259206

24. Jeong SH, Lee KM, Lee J, Bae IK, Kim JS, Kim HS, et al. Clonal and horizontal spread of the blaOXA-232 gene among Enterobacteriaceae in a Korean hospital. Diagn Microbiol Infect Dis. 2015;82(1):70-2. https://doi.org/10.1016/j. diagmicrobio.2015.02.001 PMID: 25702524

25. Yoon EJ, Kim JO, Kim D, Lee H, Yang JW, Lee KJ, et al. Klebsiella pneumoniae Carbapenemase Producers in South Korea between 2013 and 2015. Front Microbiol. 2018;9:56. https:// doi.org/10.3389/fmicb.2018.00056 PMID: 29422888

26. Kluytmans J. Plasmid-encoded colistin resistance: mcr-one, two, three and counting. Euro Surveill. 2017;22(31):30588. https://doi.org/10.2807/1560-7917.ES.2017.22.31.30588 PMID: 28797321

27. Carattoli A, Villa L, Feudi C, Curcio L, Orsini S, Luppi A, et al. Novel plasmid-mediated colistin resistance mcr-4 gene in Salmonella and Escherichia coli, Italy 2013, Spain and Belgium, 2015 to 2016. Euro Surveill. 2017;22(31):30589. https://doi.org/10.2807/1560-7917.ES.2017.22.31.30589 PMID: 28797329

28. Borowiak M, Fischer I, Hammerl IA, Hendriksen RS, Szabo I, Malorny B. Identification of a novel transposon-associated phosphoethanolamine transferase gene, mcr-5, conferring colistin resistance in d-tartrate fermenting Salmonella enterica subsp. enterica serovar Paratyphi B. J Antimicrob Chemother. 2017;72(12):3317-24. https://doi.org/10.1093/jac/dkx327 PMID: 28962028

29. Kim ES, Chong YP, Park SJ, Kim MN, Kim SH, Lee SO, et al. Detection and genetic features of MCR-1-producing plasmid in human Escherichia coli infection in South Korea. Diagn Microbiol Infect Dis. 2017;89(2):158-6o. https://doi. org/10.1016/j.diagmicrobio.2017.06.020 PMID: 28780246

30. Hong JS, Yoon EJ, Lee H, Jeong SH, Lee K. Clonal dissemination of Pseudomonas aeruginosa sequence type 235 isolates carrying blaIMP- 6 and emergence of blaGES-24 and blaIMP-10 on novel genomic islands PAGI-15 and -16 in South Korea. Antimicrob Agents Chemother. 2016;60(12):7216-23. PMID: 27671068
31. Köhler T, Michea-Hamzehpour M, Epp SF, Pechere JC. Carbapenem activities against Pseudomonas aeruginosa: respective contributions of OprD and efflux systems. Antimicrob Agents Chemother. 1999;43(2):424-7. https://doi. org/10.1128/AAC.43.2.424 PMID: 9925552

32. Jiang M, Liu L, Ma Y, Zhang Z, Li N, Zhang F, et al. Molecular epidemiology of multi-drug resistant Acinetobacter baumanni isolated in Shandong, China. Front Microbiol. 2016;7:1687. https://doi.org/10.3389/fmicb.2016.01687 PMID: 27818659

33. Yoon EJ, Kim JO, Yang JW, Kim HS, Lee KJ, Jeong SH, et al. The blaOXA-23-associated transposons in the genome of Acinetobacter spp. represent an epidemiological situation of the species encountering carbapenems. J Antimicrob Chemother. 2017;72(10):2708-14. https://doi.org/10.1093/jac/ dkx205 PMID: 29091183

\section{License and copyright}

This is an open-access article distributed under the terms of the Creative Commons Attribution (CC BY 4.0) Licence. You may share and adapt the material, but must give appropriate credit to the source, provide a link to the licence, and indicate if changes were made.

This article is copyright of the authors, 2018. 